$\mathrm{BMB}$

BMB Rep. 2017; 50(1): 1-2

Reports

www.bmbreports.org

Perspective

\title{
Interacting network of Hippo, Wnt/ $\beta$-catenin and Notch signaling represses liver tumor formation
}

\author{
Wantae Kim, Sanjoy Kumar Khan E Yingzi Yang * \\ Department of Developmental Biology, Harvard School of Dental Medicine, MA 02115, USA
}

\begin{abstract}
Acquiring a selective growth advantage by breaking the proliferation barrier established by gatekeeper genes is a centrally important event in tumor formation. Removal of the mammalian Hippo kinase Mst1 and Mst2 in hepatocytes leads to rapid hepatocellular carcinoma (HCC) formation, indicating that the Hippo signaling pathway is a critical gatekeeper that restrains abnormal growth in hepatocytes. By rigorous genetic approaches, we identified an interacting network of the Hippo, Wnt/ $\beta$-catenin and Notch signaling pathways that control organ size and HCC development. We found that in hepatocytes, the loss of Mst1/2 leads to the activation of Notch signaling, which forms a positive feedback loop with Yap/Taz (transcription factors controlled by Mst1/2). This positive feedback loop results in severe liver enlargement and rapid HCC formation. Blocking the Yap/Taz-Notch positive feedback loop by Notch inhibition in vivo significantly reduced the Yap/Taz activities, hepatocyte proliferation and tumor formation. Furthermore, we uncovered a surprising inhibitory role of $\mathrm{Wnt} / \beta$-catenin signaling to Yap/Taz activities, which are important in tumor initiation. Genetic removal of $\beta$-catenin in the liver of the Mst1/2 mutants significantly accelerates tumoriogenesis. Therefore, $\mathrm{Wnt} / \beta$-catenin signaling, known for its oncogenic property, exerts an unexpected function in restricting Yap/Taz and Notch activities in HCC initiation. The molecular interplay between the three signaling pathways identified in our study provides new insights in developing novel therapeutic strategies to treat liver tumors. [BMB Reports 2017; 50(1): 1-2]
\end{abstract}

*Corresponding author. E-mail: yingzi_yang@hsdm.harvard.edu

https://doi.org/10.5483/BMBRep.2017.50.1.196

Received 22 November 2016

Keywords: HCC, Hippo, Notch pathways, Wnt/ $\beta$-catenin, Yap/Taz

Abbreviations: HCC, Hepatocellular carcinoma; YAP, Yes-assocaited protein; Mst1/2, mammalian sterile 20-like kinase 1 and 2; NICD, notch intracellular domain

Perspective to: Kim et al (2017) Hippo signaling interactions with Wnt/ $\beta$-catenin and Notch signaling repress liver tumorigenesis. J Clin Invest. 2017 Jan 3;127(1):137-152. doi: 10.1172/JCI88486.
The liver has a remarkable regeneration property after an injury, and is able to adjust to its original size (El-Serag, N Engl J Med 2011, 365:1118-1127). The mechanism of how the liver accomplishes such capabilities has remained elusive, and is one of the most sought after answer. HCC is the fifth most frequent malignant cancer, and the second leading cause of cancer-related deaths worldwide (Torre et al., CA: a cancer journal for clinician 2012, 65:87-108). There are several risk factors, including genetic mutations, long-term virus infection and cirrhosis, that predisposes to HCC (Forner et al., Lancet 2012, 379:1245-1255). Therefore, there is a need for uncovering the appropriate molecular targets and developing new therapeutic strategies to improve patient survival. Understanding genetic and molecular lesions that cause liver cancer progression could provide fundamental insights for clinical applications.

A recently discovered Hippo signaling plays an essential role in organ size control and tumor suppression (Dong et al., Cell 2007, 130:1120-1133). Several evidences report that misregulation of Hippo signaling or Yap/Taz (the transcription factors controlled by Hippo kinases Mst1/2) is involved in diverse human diseases, including liver cancer (Song et al., Proc Natl Acad Sci USA 2010, 26:1431-1436). Recent reports suggest that approximately $30 \%$ of HCCs exhibit inactivation of tumor suppressor Mst1 and Mst2, and subsequently high Yap/Taz activity, indicating the contribution of Hippo in HCC (Zhou et al., Cancer Cell 2009, 16:425-438).

Wnts are secreted proteins that regulate many important developmental and physiological processes. $\beta$-catenin is a centrally important transcription co-activator that activates the Wnt target gene expression in the $\mathrm{Wnt} / \beta$-catenin pathway (Kim et al., Biochem J 2013, 15:9-21). Abnormal activation of the $\mathrm{Wnt} / \beta$-catenin pathway causes many types of tumors in humans, including hepatoblastoma and HCC (de La Coste et al., Proc Natl Acad Sci USA 1998, 95:8847-51). The Wnt and Hippo signaling pathways interact differentially in distinct cell compartments. In the nucleus, YAP co-operates with $\beta$-catenin to regulate the gene expression in controlling the heart size, and tumor transformation and maintenance (Heallen et al., Science 2011, 332:458-461). However, YAP/TAZ also inhibits the $\mathrm{Wnt} / \beta$-catenin activities by regulating Dishevelled (Dvl) phosphorylation and nuclear localization of Dvl or $\beta$-catenin in the cytosol (Hansen et al., Trends Cell Biol 2015, 25:

ISSN: 1976-670X (electronic edition)

Copyright (c) 2017 by the The Korean Society for Biochemistry and Molecular Biology

(c) This is an open-access article distributed under the terms of the Creative Commons Attribution Non-Commercial License (http://creativecommons.org/licenses/by-nc/4.0) which permits unrestricted non-commercial use, distribution, and reproduction in any medium, provided the original work is properly cited. 
$\longrightarrow$ Negative Feedback

$\longrightarrow$ Positive Feedback

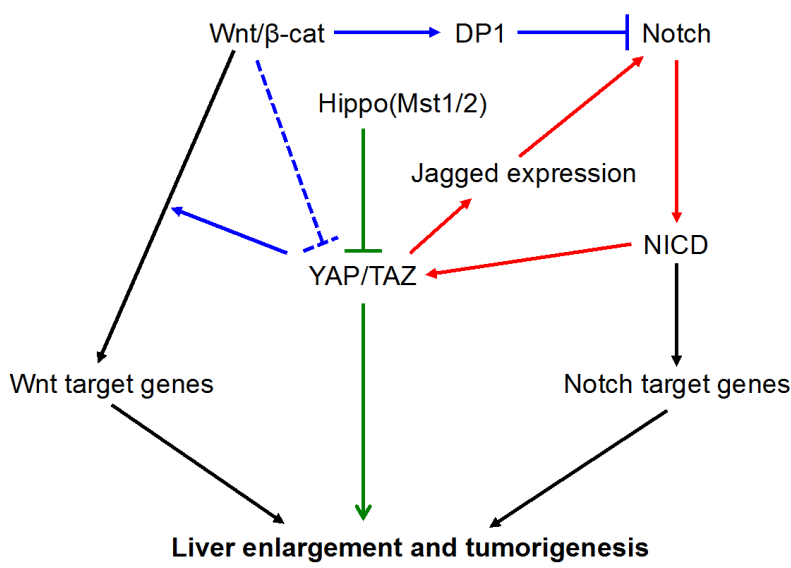

Fig. 1. Yap/Taz forms a critical positive feedback loop with Notch signaling to promote liver enlargement and turmorigenesis (red). Breaking this positive feedback-loop led to reduced hepatomegaly and tumor progression. The inhibitory role of $\mathrm{Wnt} / \beta$-catenin in the liver tumor, caused by the viscous positive feedback, is at least in part through the DP1-mediated inhibition of Notch signaling (blue).

499-513). It is as yet unknown whether the mode of interaction between YAP/TAZ and the $\mathrm{Wnt} / \beta$-catenin signaling pathways is specific to the cell type. Ours, as well as a few previous studies, have found that the loss of Mst $1 / 2$ in hepatocytes leads to tumor formation and activation of $\mathrm{Wnt} / \beta$-catenin signaling in the liver, but the role of activated $\mathrm{Wnt} / \beta$-catenin signaling in liver enlargement and tumor formation caused by inactivation of the Hippo pathway remains unknown.

The Notch signaling pathway is also critically important in both liver development and tumor formation. Notch signaling promotes formation of the oval cell, which is the liver stem cell (Tanimizu and Mitaka, Organogenesis 2014, 10:208-2015). Notch receptor and ligand expression are highly regulated, and Notch signaling is activated in HCC patients (Geisler and Strazzabosco, Hepatology 2015, 61:382-92). In mice, constitutively expressed NICD (Notch intracellular domain) in liver causes liver tumor formation (Zender et al., Cancer Cell
2013, 23:784-795). The Notch pathway is activated by direct cell-to-cell contact, which allows direct binding of Notch receptors and their membrane-bound ligands (Jagged and Delta-like). Notch ligand binding induces sequential proteolytic cleavage of Notch receptors by the ADAM family and $\gamma$-secretase complex. As a result, NICD is generated and enters the nucleus, where it forms ternary complex with co-factor RBP-j and mastermind to participate in the transcriptional regulation of target genes.

In the current study, we found that the loss of Mst $1 / 2$ in hepatocytes led to the activation of Notch signaling, which forms a positive feedback loop with Yap/Taz. This positive feedback loop resulted in severe liver enlargement and rapid HCC formation. Furthermore, we uncovered a surprising inhibitory role of $\mathrm{Wnt} / \beta$-catenin signaling to $\mathrm{Yap} / \mathrm{Taz}$ activities that is important in tumor initiation. Genetic removal of $\beta$-catenin in the liver of the Mst $1 / 2$ null mutants significantly increased the number of tumor nodules, that also appeared at younger ages. Mechanistically, we have identified that increased generation of the Notch intracellular domain (NICD) by Yap/Taz activation also stabilized Taz by inhibiting its binding to $\beta$-TrCP. Wnt $\beta$-catenin signaling suppressed the positive feedback loop between Notch and Taz through promoting the nuclear localization of DP1 (the dimerization partner of E2F transcriptional factors) which subsequently inhibits Notch activity. Breaking down the Yap/Taz-Notch positive feedback loop by Notch inhibition in vivo, significantly reduced Yap/Taz activities, hepatocyte proliferation and tumor formation. Therefore, $W \mathrm{nt} / \beta$-catenin signaling, which is known for its oncogenic property, exerts an unexpected function in restricting Yap/Taz and Notch activities in HCC initiation. Overall, our results uncovered a molecular interplay between the three signaling pathways, and our studies have shed new insights on developing therapeutic strategies to treat liver tumors (Fig. 1).

\section{ACKNOWLEDGEMENTS}

This study is supported by the intramural research program of National Human Genome Research Institute (NHGRI) and Harvard School of Dental Medicine. WK (HI13C1274) is supported by the Korean Visiting Scientist Training Award (KVSTA) fellowship from Korea Health Industry Development Institute (KHIDI). 